\title{
Advances in neonatal screening for primary immune deficiencies (Review)
}

\author{
TINGTING JIANG, ZHENGUANG LI and QIULI ZHANG \\ Department of Neonatology, Xuzhou Children's Hospital, Xuzhou, Jiangsu 221002, P.R. China
}

Received November 13, 2015; Accepted March 1, 2016

DOI: $10.3892 / e t m .2016 .3119$

\begin{abstract}
The congenital disorders of immune competence are known as primary immunodeficiencies (PID) and are mainly characterized by a pathological susceptibility to infection. These infections are mostly of time repetitive and drug resistant in nature. The number of infected infants has reached over 200 and is on the increase. Additionally, clinical severity of the disease has been confirmed to be extensive. The increasing number of these severe PIDs is due to the lack of specific as well as efficient management avenues. New assays and concepts for newborn screening of severe primary immune deficiencies are being explored and the present review focused on these new upcoming strategies for improved screening of neonates.
\end{abstract}

\section{Contents}

1. Introduction

2. Neonatal screening and its importance

3. DNA-based newborn screening assays for severe PID: Overview and updates

4. Immunophenotype screening in premature newborns

5. Genetic confirmation of PIDs

6. Latest advances

\section{Introduction}

The conciliated ability to fight infections in the environment due to exposure to chemotherapy, radiation, glucocorticoids and anti-rheumatic drugs, malnutrition, aging, malignancies or metabolic diseases is the major hallmark of acquired immune deficiency. However, the inability to fight infections right from birth is termed primary immunodeficiencies (PID)

Correspondence to: Zhenguang Li, Department of Neonatology, Xuzhou Children's Hospital, 18 Sudibei Road, Xuzhou, Jiangsu 221002, P.R. China

E-mail: zhenguangli29@163.com

Key words: primary immunodeficiencies, neonate screening, immune competence and these are genetically determined disorders of protective immune function. At present, over 240 distinct entities of PIDs are known and are present as phagocytic disorders, complement deficiencies, T-cell deficiencies and, predominantly, antibody disorders (1). Despite scientific advancements in the field, patients with primary antibody deficiencies in particular are prone to a significant diagnostic delay, thereby promoting an increased likelihood to develop and progress infection-associated organ damage (2,3). For instance, chronic lung disease, characterized by moderate to severe bronchiectasis observed in these patients, remains an unresolved threat to the overall life expectancy despite virtually normal levels of serum immunoglobulin $\mathrm{G}$ following replacement therapy $(4,5)$. Clinical experience endorses disease awareness and should guide investigations into newborn screening, disease progression and future therapeutic strategies (6).

PID are not rare diseases and should be considered in all patients with severe or recurrent infections admitted to health care centres. To reduce significant morbidity associated with antibody deficiency diseases and to improve patient quality-of-life and the overall survival, it is crucial that strategies be employed that would allow earliest-possible diagnosis of these patients. Such strategies include means of newborn screening, and prompt initiation of immunoglobulin replacement therapy as well as further supportive care. The present review article focused on the latest updates in the field of neonatal screening for primary immunodeficiency disorders.

\section{Neonatal screening and its importance}

Case procedures already anticipate the patient-centred approach that is employed at present to identify primary immunodeficiency diseases based on warning signs circularization and clinical pattern education to alleviate or prevent the impact of a delayed diagnosis (7). In addition, sophisticated diagnostic tools such as molecular assays suitable for high throughput screening of newborns have been identified in the PID field and are currently undergoing evaluation (7). Furthermore, a modern management of patients requiring substitution of immunoglobulin products, including those diagnosed with (severe) combined immunodeficiencies, primary B-cell immunodeficiencies and selected disorders such as the Wiskott-Aldrich syndrome, has advanced to provide a more sustainable therapeutic effect and to improve the patients' quality-of-life 
than merely replacing antibody protection $(8,9)$. The summarized prevalence of PIDs ranges from 1/500 to 1/2.000 of the general population; however, PID are misdiagnosed in many cases because of their prolonged course of disease which often worsens the outcome. Severe forms of PID require immediate treatment ranges from 2 to 8/100.000 live births, making high demands on the effectiveness and availability of diagnostic tests to detect them as early as possible. The purpose of neonatal testing is the early recognition of treatable, mostly genetically determined diseases that manifest with a high rate of morbidity and mortality. The earliest possible diagnosis of severe PID improves their prognosis and treatment efficiency significantly (10). This stems primarily from the possibility to initiate early preventive measures to avoid infection, and to prevent iatrogenic damage, such as that caused by the administration of recommended vaccinations, including the rotavirus vaccine (live vaccine), which is safe for immunocompetent infants (11). The prevention of such complications significantly improves overall survival prior to and after performing curative hematopoietic stem cell transplantation (HSCT), gene therapy (GT) or supportive enzyme replacement therapy or immunoglobulin replacement therapy.

\section{DNA-based newborn screening assays for severe PID: Overview and updates}

The requirements for screening markers for severe PID are usually more demanding. Severe combined immunodeficiencies (SCID) and XLA are pathogenetically and immunophenotypically characterized by the absence of $\mathrm{T}$ and/or B lymphocytes. In the evaluation of different laboratory methods for the detection of these PID, the measurement of episomal excision products of lymphocyte receptors (TRECs and KRECs) has prevailed, as this method, both in terms of test quality and practical feasibility, is consistent with prospective screenings (12). When applying the evaluation criteria of population-based screening tests to the measurement of TRECs and KRECs for the detection of the pronounced neonatal deficiency of autologous $\mathrm{T}$ and/or B lymphocytes, the implementation of newborn screening has been viewed positively (13).

The principle of measuring episomal excision products of lymphocyte receptors is based on naturally occurring recombination and affinity maturation of the T-cell and B-cell receptors (14). Sections are excised from the germline DNA of immunoglobulin genes that are not involved in the recombination process of the antigen receptors. While $\mathrm{T}$ lymphocytes in the thymus initially excise portions of the $\delta$-locus in order to then recombine the $\alpha$-locus, so-called TRECs are created, which spontaneously form circular DNA fragments that are not replicated further during cell division. Following a similar principle, a deletion occurs in B lymphocytes in the bone marrow during the $\mathrm{Vk}-\mathrm{Jk}$ rearrangement, in the process of which KRECs are formed. A recent study utilized a uniform PCR strategy to determine the presence and number of copies of TRECs and KRECs in dried blood samples from neonates (15). This has resulted in development of a TREC-KREC screening test that offers good resolution for low copy numbers, allowing for the implementation of a diagnostic threshold defined by the user in the screening laboratory, thus making it possible to differentiate 'typical' SCID and XLA patients from those with borderline screening results observed in profound combined immunodeficiency diseases.

\section{Immunophenotype screening in premature newborns}

Immunophenotyping often fails to provide a definitive assessment of the functional relevance of an anticipated severe PID, especially when the flow cytometric analysis or clinical examination suggests the existence of an 'atypical' phenotype. Therefore, for the completion of flow cytometric tests, i) the lymphocytic proliferation and stimulation ability, as well as ii) the functionality of the innate diversity of the immune repertoire should also be examined in vitro (16). Infants born before 37 weeks of gestation may suffer from a variety of cardiovascular, neurological, metabolic and gastrointestinal complications, in addition to an increased susceptibility to respiratory and urinary tract infections (17). Although many risk factors have been linked to preterm birth, the multi-factorial causes remain unresolved in the majority of affected infants, limiting the availability of effective interventions. In developed countries, the birth rate of premature newborns is $5-12 \%$ with an increasing trend, posing concern for newborn screening programs in particular and for public healthcare systems in general. Thus, if dried blood spot samples are taken prior to the 32nd week of gestation, a second analysis conducted subsequently for newborn screening tests of metabolic disorders to distinguish abnormal results of genetic origin from physiological immaturity is useful in the timely detection of PIDs (17). Thus, this approach is clearly beneficial in the timely diagnosis of PIDs which in turn, results in improved treatment planning. However, confirmatory genetic testing is also essential after immunophenotyping to confirm the presence of PIDs and is therefore discussed below.

\section{Genetic confirmation of PIDs}

Over 18 monogenetic defects have been described, resulting in a phenotype of SCID (18). Similarly, primary agammaglobulinemia with some missing B-lymphocytes can be traced back to more than six genetic defects (1). In terms of pathogenesis, the underlying defects can be associated with maturation disorders, especially of the lymphatic lineage in the bone marrow, as well as in primary and secondary lymphatic tissues. Flow cytometric evaluation of the peripheral blood makes it possible for SCID patients to be classified according to the $\mathrm{T}-\mathrm{B}+/-\mathrm{NK}+/-$ system, which indicates the underlying genetic defect (1). The molecular and cell functional heterogeneity of SCID is also evident in the clinical treatment experience, which led to the application of 'gene-specific' protocols in conditioning and stem cell transplantation or GT for SCID patients $(19,20)$.

Decision making with regard to stem cell transplantation has been based on the clinical course, as well as cellular and immunofunctional tests. However, the presymptomatic early identification of newborns with severe PID poses a new challenge for the algorithms of treatment decision. Immunological function testing of infants, in particular, is challenged by the lack of sufficient reference ranges, because age-weighted control samples are not readily available. 
Therefore, molecular genetic tests are of particular importance for newborns with neonatal deficiency of autologous $\mathrm{T}$ lymphocytes and a suspected SCID or PCID phenotype. At the same time, the initiation of a curative therapy should not be delayed, by either the time required for molecular testing, or by the absence of a final genetic diagnosis. In newborns with severe neonatal deficiency of autologous B lymphocytes and suspicion of agammaglobulinemia transferred maternal immunity, which usually lasts up to 6 months following birth, it can help to sustain the time until a complete genetic diagnosis prior to initiation of treatment is made (21).

\section{Latest advances}

At present, molecular genetic testing in a clinical context is predominantly performed through the selection of annotated candidate genes for the suspected congenital immunodeficiency. However, the limitation to candidate genes is an issue with regard to the molecular heterogeneity and known overlapping effects in the clinical phenotype, especially in the case of a sequential approach ('gene-by-gene'). Recent molecular genetic methods can partially or completely overcome these limitations; for example, DNA microarrays, allele-specific PCR, allele-specific primer extension, PCR oligonucleotide ligation and hybridization methods including ligation PCR $(22,23)$. However, sequence-analytical challenges in view of ever increasing amounts of data are currently the primary obstacle to a timely reporting of results. On the other hand, the latest generation of sequencers suggests a cost- and time-efficient analysis of entire transcriptomes, exomes or even genomes (24). The advantage of transcriptome or genome-wide tests is the ability to simultaneously examine comprehensively known candidate genes sequentially and to identify new disease-associated genes for PID. In view of the evidence-based justification for lifelong supportive or invasive therapies, such as HSCT, this is likely to become increasingly important (25).

In conclusion, investigations into making diagnosis of PIDs specific and effective as well as novel diagnostic assays to be used for newborn screening of severe PID are ongoing.

\section{References}

1. Al-Herz W, Bousfiha A, Casanova JL, Chatila T, Conley ME, Cunningham-Rundles C, Etzioni A, Franco JL, Gaspar HB, Holland SM, et al: Primary immunodeficiency diseases: An update on the classification from the international union of immunological societies expert committee for primary immunodeficiency. Front Immunol 5: 162, 2014

2. Gathmann B, Binder N, Ehl S and Kindle G; ESID Registry Working Party: The European internet-based patient and research database for primary immunodeficiencies: Update 2011 Clin Exp Immunol 167: 479-491, 2012.

3. Cunningham-Rundles C: Lung disease, antibodies and other unresolved issues in immune globulin therapy for antibody deficiency. Clin Exp Immunol 157 (Suppl 1): 12-16, 2009.

4. Loebinger MR, Wells AU, Hansell DM, Chinyanganya N, Devaraj A, Meister M and Wilson R: Mortality in bronchiectasis: A long-term study assessing the factors influencing survival. Eur Respir J 34: 843-849, 2009.

5. Aghamohammadi A, Allahverdi A, Abolhassani H, Moazzami K, Alizadeh H, Gharagozlou M, Kalantari N, Sajedi V, Shafiei A, Parvaneh N, et al: Comparison of pulmonary diseases in common variable immunodeficiency and X-linked agammaglobulinaemia. Respirology 15: 289-295, 2010.
6. Borte S, Lanig H, Borte M, Fasshauer M and Sack U: Therapeutic implications of the IL-21: IL-4 receptor system in children with common variable immunodeficiency syndrome. Klin Padiatr 222: 362-367, 2010.

7. Chan K, Davis J, Pai SY, Bonilla FA, Puck JM and Apkon M: A Markov model to analyze cost-effectiveness of screening for severe combined immunodeficiency (SCID). Mol Genet Metab 104: 383-389, 2011.

8. Orange JS, Belohradsky BH, Berger M, Borte M, Hagan J, Jolles S, Wasserman RL, Baggish JS, Saunders R and Grimbacher B: Evaluation of correlation between dose and clinical outcomes in subcutaneous immunoglobulin replacement therapy. Clin Exp Immunol 169: 172-181, 2012.

9. Kaveri SV, Maddur MS, Hegde P, Lacroix-Desmazes S and Bayry $\mathrm{J}$ : Intravenous immunoglobulins in immunodeficiencies: More than mere replacement therapy. Clin Exp Immunol 164 (Suppl 2): 2-5, 2011.

10. Brown L, Xu-Bayford J, Allwood Z, Slatter M, Cant A, Davies EG, Veys P, Gennery AR and Gaspar HB: Neonatal diagnosis of severe combined immunodeficiency leads to significantly improved survival outcome: The case for newborn screening. Blood 117: 3243-3246, 2011.

11. Bakare N, Menschik D, Tiernan R, Hua W and Martin D: Severe combined immunodeficiency (SCID) and rotavirus vaccination: Reports to the Vaccine Adverse Events Reporting System (VAERS). Vaccine 28: 6609-6612, 2010.

12. Puck JM: Laboratory technology for population-based screening for severe combined immunodeficiency in neonates: The winner is T-cell receptor excision circles. J Allergy Clin Immunol 129: 607-616, 2012

13. Borte S, von Döbeln U and Hammarström L: Guidelines for newborn screening of primary immunodeficiency diseases. Curr Opin Hematol 20: 48-54, 2013.

14. van Zelm MC, van der Burg M, Langerak AW and van Dongen JJ: PID comes full circle: Applications of V(D)J recombination excision circles in research, diagnostics and newborn screening of primary immunodeficiency disorders. Front Immunol 2: 12 , 2011.

15. Somech R and Etzioni A: A call to include severe combined immunodeficiency in newborn screening program. Rambam Maimonides Med J 5: e0001, 2014.

16. Borte S, Wang N, Oskarsdóttir S, von Döbeln U and Hammarström L: Newborn screening for primary immunodeficiencies: Beyond SCID and XLA. Ann N Y Acad Sci 1246: 118-130, 2011.

17. Speckmann C, Neumann C, Borte S, la Marca G, Sass JO, Wiech E, et al: Delayed-onset adenosine deaminase deficiency: strategies for an early diagnosis. J Allergy Clin Immunol 130: 991- 994, 2012

18. van der Burg M and Gennery AR: Educational paper. The expanding clinical and immunological spectrum of severe combined immunodeficiency. Eur J Pediatr 170: 561-571, 2011.

19. Hönig M, Schulz A and Friedrich W: Hematopoietic stem cell transplantation for severe combined immunodeficiency. Klin Padiatr 223: 320-325, 2011.

20. Cavazzana-Calvo M, Fischer A, Hacein-Bey-Abina S and Aiuti A: Gene therapy for primary immunodeficiencies: Part 1. Curr Opin Immunol 24: 580-584, 2012.

21. Okocha IU, Hanson CG, Chinen J and Shearer WT: Decline of antibodies in XLA infant: When to start IVIG. Allergy 66: 434-435, 2011.

22. Chen CH, Hsu CL, Huang SH, Chen SY, Hung YL, Chen HR, Wu YC, Su LJ and Lee HC: Method designed to respect molecular heterogeneity can profoundly correct present data interpretations for genome-wide expression analysis. PLoS ONE 10: March 20, 2015 (Epub ahead of print). doi:10.1371/journal.pone.0121154.

23. Russo VEA, Brody S, Cove D and Ottolenghi S: Development: The Molecular Genetic Approach. Springer Science \& Business Media, p.605, 2012

24. Ghosh S, Krux F, Binder V, Gombert M, Niehues T, Feyen O, Laws HJ and Borkhardt A; PID-NET: German Network on Primary Immunodeficiency Diseases: Array-based sequence capture and next-generation sequencing for the identification of primary immunodeficiencies. Scand J Immunol 75: 350-354, 2012.

25. Chou J, Ohsumi TK and Geha RS: Use of whole exome and genome sequencing in the identification of genetic causes of primary immunodeficiencies. Curr Opin Allergy Clin Immunol 12: 623-628, 2012. 